\title{
Measurement of the $b$ Hadron Lifetime with the Dipole Method
}

\author{
The ALEPH Collaboration
}

\begin{abstract}
A measurement of the average lifetime of $b$ hadrons has been performed with the dipole method on a sample of 260,000 hadronic $Z$ decays recorded with the ALEPH detector during 1991. The dipole is the distance between the vertices built in the opposite hemispheres. The mean dipole is extracted from all the events without attempting $b$ enrichment. Comparing the average of the data dipole distribution with a Monte Carlo calibration curve obtained with different $b$ lifetimes, an average $b$ hadron lifetime of $1.51 \pm 0.08 \mathrm{ps}$ is extracted.
\end{abstract}





\section{The ALEPH Collaboration}

D. Buskulic, I. De Bonis, D. Decamp, P. Ghez, C. Goy, J.-P. Lees, M.-N. Minard, B. Pietrzyk Laboratoire de Physique des Particules (LAPP), IN ${ }^{2} P^{3}$-CNRS, 74019 Annecy-le-Vieux Cedex, France

F. Ariztizabal, P. Comas, J.M. Crespo, M. Delfino, I. Efthymiopoulos, E. Fernandez, M. Fernandez-Bosman,

V. Gaitan, Ll. Garrido, T. Mattison, A. Pacheco, C. Padilla, A. Pascual Institut de Fisica d'Altes Energies, Universitat Autonoma de Barcelona, 08193 Bellaterra (Barcelona), $\operatorname{Spain}^{7}$

D. Creanza, M. de Palma, A. Farilla, G. Iaselli, G. Maggi, S. Natali, S. Nuzzo, M. Quattromini, A. Ranieri, G. Raso, F. Romano, F. Ruggieri, G. Selvaggi, L. Silvestris, P. Tempesta, G. Zito INFN Sezione di Bari e Dipartimento di Fisica dell' Università, 70126 Bari, Italy

Y. Chai, H. Hu, D. Huang, X. Huang, J. Lin, T. Wang, Y. Xie, D. Xu, R. Xu, J. Zhang, L. Zhang, W. Zhao Institute of High-Energy Physics, Academia Sinica, Beijing, The People's Republic of China ${ }^{8}$

E. Blucher, ${ }^{22}$ G. Bonvicini, J. Boudreau, D. Casper, H. Drevermann, R.W. Forty, G. Ganis, C. Gay, R. Hagelberg, J. Harvey, J. Hilgart, ${ }^{31}$ R. Jacobsen, B. Jost, J. Knobloch, I. Lehraus, T. Lohse, ${ }^{27}$ M. Maggi, C. Markou, M. Martinez, P. Mato, H. Meinhard, A. Minten, A. Miotto, R. Miquel, H.-G. Moser, P. Palazzi, J.R. Pater, J.A. Perlas, J.-F. Pusztaszeri, F. Ranjard, G. Redlinger, ${ }^{23}$ L. Rolandi, J. Rothberg, ${ }^{2}$ T. Ruan, M. Saich, D. Schlatter, M. Schmelling, F. Sefkow, ${ }^{6}$ W. Tejessy, I.R. Tomalin, R. Veenhof, H. Wachsmuth, S. Wasserbaech, ${ }^{2}$ W. Wiedenmann, T. Wildish, W. Witzeling, J. Wotschack European Laboratory for Particle Physics (CERN), 1211 Geneva 23, Switzerland

Z. Ajaltouni, F. Badaud, M. Bardadin-Otwinowska, R. El Fellous, A. Falvard, P. Gay, C. Guicheney, P. Henrard, J. Jousset, B. Michel, J-C. Montret, D. Pallin, P. Perret, F. Podlyski, J. Proriol, F. Prulhière, F. Saadi Laboratoire de Physique Corpusculaire, Université Blaise Pascal, IN ${ }^{2} P^{3}$-CNRS, Clermont-Ferrand, 63177 Aubière, France

T. Fearnley, J.B. Hansen, J.D. Hansen, J.R. Hansen, ${ }^{1}$ P.H. Hansen, R. Møllerud, B.S. Nilsson ${ }^{1}$ Niels Bohr Institute, 2100 Copenhagen, Denmark ${ }^{9}$

A. Kyriakis, E. Simopoulou, I. Siotis, A. Vayaki, K. Zachariadou Nuclear Research Center Demokritos (NRCD), Athens, Greece

J. Badier, A. Blondel, G. Bonneaud, J.C. Brient, G. Fouque, S. Orteu, A. Rougé, M. Rumpf, R. Tanaka, M. Verderi, H. Videau Laboratoire de Physique Nucléaire et des Hautes Energies, Ecole Polytechnique, IN ${ }^{2} P^{3}{ }_{-}$CNRS, 91128 Palaiseau Cedex, France

D.J. Candlin, M.I. Parsons, E. Veitch Department of Physics, University of Edinburgh, Edinburgh EH9 3JZ, United Kingdom ${ }^{10}$

E. Focardi, L. Moneta, G. Parrini Dipartimento di Fisica, Università di Firenze, INFN Sezione di Firenze, 50125 Firenze, Italy

M. Corden, C. Georgiopoulos, M. Ikeda, D. Levinthal ${ }^{15}$ Supercomputer Computations Research Institute and Dept. of Physics, Florida State University, Tallahassee, FL 32306, USA $12,13,14$

A. Antonelli, R. Baldini, G. Bencivenni, G. Bologna, ${ }^{4}$ F. Bossi, P. Campana, G. Capon, F. Cerutti, V. Chiarella, B. D’Ettorre-Piazzoli, ${ }^{24}$ G. Felici, P. Laurelli, G. Mannocchi, ${ }^{5}$ F. Murtas, G.P. Murtas, L. Passalacqua, M. PepeAltarelli, P. Picchi ${ }^{4}$ Laboratori Nazionali dell'INFN (LNF-INFN), 00044 Frascati, Italy 
P. Colrain, I. ten Have, J.G. Lynch, W. Maitland, W.T. Morton, C. Raine, P. Reeves, J.M. Scarr, K. Smith, M.G. Smith, A.S. Thompson, R.M. Turnbull

Department of Physics and Astronomy, University of Glasgow, Glasgow G12 8QQ, United Kingdom ${ }^{10}$

B. Brandl, O. Braun, C. Geweniger, P. Hanke, V. Hepp, E.E. Kluge, Y. Maumary, A. Putzer, B. Rensch, A. Stahl, K. Tittel, M. Wunsch

Institut für Hochenergiephysik, Universität Heidelberg, 6900 Heidelberg, Fed. Rep. of Germany ${ }^{16}$

R. Beuselinck, D.M. Binnie, W. Cameron, M. Cattaneo, D.J. Colling, P.J. Dornan, A.M. Greene, J.F. Hassard, N.M. Lieske, ${ }^{29}$ A. Moutoussi, J. Nash, S. Patton, D.G. Payne, M.J. Phillips, G. San Martin, J.K. Sedgbeer, A.G. Wright

Department of Physics, Imperial College, London SW7 2BZ, United Kingdom ${ }^{10}$

P. Girtler, D. Kuhn, G. Rudolph, R. Vogl

Institut für Experimentalphysik, Universität Innsbruck, 6020 Innsbruck, Austria ${ }^{18}$

C.K. Bowdery, T.J. Brodbeck, A.J. Finch, F. Foster, G. Hughes, D. Jackson, N.R. Keemer, M. Nuttall, A. Patel, T. Sloan, S.W. Snow, E.P. Whelan

Department of Physics, University of Lancaster, Lancaster LA1 4YB, United Kingdom ${ }^{10}$

K. Kleinknecht, J. Raab, B. Renk, H.-G. Sander, H. Schmidt, F. Steeg, S.M. Walther, R. Wanke, B. Wolf Institut für Physik, Universität Mainz, 6500 Mainz, Fed. Rep. of Germany ${ }^{16}$

A.M. Bencheikh, C. Benchouk, A. Bonissent, J. Carr, P. Coyle, J. Drinkard, ${ }^{3}$ F. Etienne, D. Nicod, S. Papalexiou, P. Payre, L. Roos, D. Rousseau, P. Schwemling, M. Talby

Centre de Physique des Particules, Faculté des Sciences de Luminy, IN ${ }^{2} P^{3}$-CNRS, 13288 Marseille,

France

S. Adlung, R. Assmann, C. Bauer, W. Blum, D. Brown, P. Cattaneo, ${ }^{26}$ B. Dehning, H. Dietl, F. Dydak, ${ }^{21}$ M. Frank, A.W. Halley, K. Jakobs, J. Lauber, G. Lütjens, G. Lutz, W. Männer, R. Richter, J. Schröder, A.S. Schwarz,

R. Settles, H. Seywerd, U. Stierlin, U. Stiegler, R. St. Denis, G. Wolf

Max-Planck-Institut für Physik, Werner-Heisenberg-Institut, 8000 München, Fed. Rep. of Germany ${ }^{16}$

R. Alemany, J. Boucrot, ${ }^{1}$ O. Callot, A. Cordier, M. Davier, L. Duflot, J.-F. Grivaz, Ph. Heusse, D.E. Jaffe, P. Janot, D.W. Kim ${ }^{19}$ F. Le Diberder, J. Lefrançois, A.-M. Lutz, M.-H. Schune, J.-J. Veillet, I. Videau, Z. Zhang Laboratoire de l'Accélérateur Linéaire, Université de Paris-Sud, IN ${ }^{2} P^{3}$ CNRS, 91405 Orsay Cedex, France

D. Abbaneo, G. Bagliesi, G. Batignani, U. Bottigli, C. Bozzi, G. Calderini, M. Carpinelli, M.A. Ciocci, V. Ciulli, R. Dell'Orso, I. Ferrante, F. Fidecaro, L. Foà, F. Forti, A. Giassi, M.A. Giorgi, A. Gregorio, F. Ligabue, A. Lusiani, E.B. Mannelli, P.S. Marrocchesi, A. Messineo, F. Palla, G. Rizzo, G. Sanguinetti, P. Spagnolo, J. Steinberger,

R. Tenchini, G. Tonelli, ${ }^{32}$ G. Triggiani, A. Valassi, C. Vannini, A. Venturi, P.G. Verdini, J. Walsh Dipartimento di Fisica dell'Università, INFN Sezione di Pisa, e Scuola Normale Superiore, 56010 Pisa, Italy

A.P. Betteridge, Y. Gao, M.G. Green, P.V. March, Ll.M. Mir, T. Medcalf, I.S. Quazi, J.A. Strong, L.R. West Department of Physics, Royal Holloway \& Bedford New College, University of London, Surrey TW20 OEX, United Kingdom ${ }^{10}$

D.R. Botterill, R.W. Clifft, T.R. Edgecock, S. Haywood, P.R. Norton, J.C. Thompson Particle Physics Dept., Rutherford Appleton Laboratory, Chilton, Didcot, Oxon OX11 OQX, United King dom ${ }^{10}$

B. Bloch-Devaux, P. Colas, H. Duarte, S. Emery, W. Kozanecki, E. Lançon, M.C. Lemaire, E. Locci, B. Marx, P. Perez, J. Rander, J.-F. Renardy, A. Rosowsky, A. Roussarie, J.-P. Schuller, J. Schwindling, D. Si Mohand, B. Vallage Service de Physique des Particules, DAPNIA, CE-Saclay, 91191 Gif-sur-Yvette Cedex, France ${ }^{17}$ 
R.P. Johnson, A.M. Litke, G. Taylor, J. Wear

Institute for Particle Physics, University of California at Santa Cruz, Santa Cruz, CA 95064, USA 25

J.G. Ashman, W. Babbage, C.N. Booth, C. Buttar, S. Cartwright, F. Combley, I. Dawson, L.F. Thompson

Department of Physics, University of Sheffield, Sheffield S3 $7 R H$, United Kingdom ${ }^{10}$

E. Barberio, A. Böhrer, S. Brandt, G. Cowan, ${ }^{1}$ C. Grupen, G. Lutters, F. Rivera, ${ }^{30}$ U. Schäfer, L. Smolik

Fachbereich Physik, Universität Siegen, 5900 Siegen, Fed. Rep. of Germany ${ }^{16}$

L. Bosisio, R. Della Marina, G. Giannini, B. Gobbo, F. Ragusa ${ }^{20}$

Dipartimento di Fisica, Università di Trieste e INFN Sezione di Trieste, 34127 Trieste, Italy

L. Bellantoni, W. Chen, J.S. Conway, ${ }^{28}$ Z. Feng, D.P.S. Ferguson, Y.S. Gao, J. Grahl, J.L. Harton, O.J. Hayes III, J.M. Nachtman, Y.B. Pan, Y. Saadi, M. Schmitt, I. Scott, V. Sharma, Z.H. Shi, J.D. Turk, A.M. Walsh, F.V. Weber, Sau Lan Wu, X. Wu, M. Zheng, G. Zobernig

Department of Physics, University of Wisconsin, Madison, WI 53706, USA ${ }^{11}$

\footnotetext{
${ }^{1}$ Now at CERN, PPE Division, 1211 Geneva 23, Switzerland.

${ }^{2}$ Permanent address: University of Washington, Seattle, WA 98195, USA.

${ }^{3}$ Now at University of California, Irvine, CA 92717, USA.

${ }^{4}$ Also Istituto di Fisica Generale, Università di Torino, Torino, Italy.

${ }^{5}$ Also Istituto di Cosmo-Geofisica del C.N.R., Torino, Italy.

${ }^{6}$ Now at DESY, Hamburg, Germany.

${ }^{7}$ Supported by CICYT, Spain.

${ }^{8}$ Supported by the National Science Foundation of China.

${ }^{9}$ Supported by the Danish Natural Science Research Council.

${ }^{10}$ Supported by the UK Science and Engineering Research Council.

${ }^{11}$ Supported by the US Department of Energy, contract DE-AC02-76ER00881.

${ }^{12}$ Supported by the US Department of Energy, contract DE-FG05-87ER40319.

${ }^{13}$ Supported by the NSF, contract PHY-8451274.

${ }^{14}$ Supported by the US Department of Energy, contract DE-FC05-85ER250000.

${ }^{15}$ Supported by SLOAN fellowship, contract BR 2703.

${ }^{16}$ Supported by the Bundesministerium für Forschung und Technologie, Fed. Rep. of Germany.

${ }^{17}$ Supported by the Direction des Sciences de la Matière, C.E.A.

${ }^{18}$ Supported by Fonds zur Förderung der wissenschaftlichen Forschung, Austria.

${ }^{19}$ Supported by the Korean Science and Engineering Foundation and Ministry of Education.

${ }^{20}$ Now at Dipartimento di Fisica, Università di Milano, Milano, Italy.

${ }^{21}$ Also at CERN, PPE Division, 1211 Geneva 23, Switzerland.

${ }^{22}$ Now at University of Chicago, Chicago, IL 60637, U.S.A.

${ }^{23}$ Now at TRIUMF, Vancouver, B.C., Canada.

${ }^{24}$ Also at Università di Napoli, Dipartimento di Scienze Fisiche, Napoli, Italy.

${ }^{25}$ Supported by the US Department of Energy, grant DE-FG03-92ER40689.

${ }^{26}$ Now at Università di Pavia, Pavia, Italy.

${ }^{27}$ Now at Max-Planck-Institut f. Kernphysik, Heidelberg, Germany.

${ }^{28}$ Now at Rutgers University, Piscataway, NJ 08854, USA.

${ }^{29}$ Now at Oxford University, Oxford OX1 3RH, U.K.

${ }^{30}$ Partially supported by Colciencias, Colombia.

${ }^{31}$ Now at SSCL, Dallas 75237-3946, TX, U.S.A.

${ }^{32}$ Also at Istituto di Matematica e Fisica, Università di Sassari, Sassari, Italy.
} 



\section{Introduction}

The average lifetime of $b$ hadrons has been measured in the last years by several experiments at PEP [1-4], PETRA [5-6], and LEP [7-10]. Most of the experiments measured the lifetime enriching the data sample with $b \bar{b}$ events through high $p, p_{T}$ leptons and fitting their impact parameter distribution. A few [5, 8] did not enrich the sample and measured the lifetime through a fit of the impact parameter distribution of high $p, p_{T}$ hadrons [8] or through the displacement of the decay vertices [5].

The measurements with no enrichment scheme have so far not been as precise as those with leptons because of the small $b \bar{b}$ fraction in hadronic events in experiments running far from the $\mathrm{Z}$ peak and the limited knowledge of the production and decay properties of the $b$ hadrons. The large fraction of $b \bar{b}$ events at the $\mathrm{Z}$ peak, the recent advance in knowledge of the $b$ quark hadronization and decay properties and the excellent tracking capability of the ALEPH detector, have made possible the measurement of the $b$ lifetime without enrichment scheme with a precision comparable to that achieved with leptons.

In the present analysis, the dipole method, pionereed in $[5,11]$, is applied on a sample of 260,000 hadronic events recorded with the ALEPH detector in 1991. It measures the distance between two vertices reconstructed in a hadronic event corresponding to two $b$ decays and compares the averages of the data and Monte Carlo distributions to extract the lifetime.

\section{The detector}

A detailed description of the ALEPH detector is given in [12]. Briefly, charged tracks are measured over the polar angle range $|\cos \theta|<0.966$ by means of an Inner Tracking Chamber (ITC) and a Time Projection Chamber (TPC). The ITC is a cylindrical drift chamber with eight axial wire layers at radii between 16 to $26 \mathrm{~cm}$. The TPC provides up to 21 space points per track at radii between 40 and $171 \mathrm{~cm}$. The tracking system is followed by a high granularity lead-proportional tube electromagnetic calorimeter (ECAL). The calorimeter is contained in a superconducting coil providing a magnetic field of $1.5 \mathrm{~T}$. The return yoke of the magnet is instrumented with streamer tubes to form a hadron calorimeter (HCAL) and is followed by two planes of streamer tubes serving as muon chambers. The calorimeters are not used explicitly in this analysis but they are used in the trigger and hadronic event selection.

Since 1991, two layers of a double sided silicon microstrip Vertex Detector (VDET) [13] have been installed between the beam pipe and the ITC at radii of 6.3 and $10.8 \mathrm{~cm}$. It provides full coverage in the azimuthal angle $\phi$. The coverage in polar angle is $|\cos \theta|<0.85$ for the inner layer and $|\cos \theta|<0.69$ for the outer. The VDET position resolution is $12 \mu \mathrm{m}$ at normal incidence for both $r$ - $\phi$ and $r-z$ [14]. Using VDET and the beam spot information, the interaction point is known on an event by event basis with an average precision of $\sigma_{y}=10 \mu \mathrm{m}$ vertically and $\sigma_{x}=60 \mu \mathrm{m}$ horizontally. The position of the beam spot center is known within $25 \mu \mathrm{m}$ in $x$ and $10 \mu \mathrm{m}$ in $y$. 


\section{The dipole method}

The dipole method is a method that makes use of all two jet hadronic events to measure the $b$ hadron lifetime.

The tracks and the event axis are projected on the $r$ - $\phi$ plane where, for each hemisphere separately, a weighted average of the intersection points of the jet tracks with the axis is calculated. The distance between these two points projected along the three-dimensional event axis is the dipole.

The analysis is performed both on the data and on a number of Monte Carlo samples generated with different lifetimes. The averages of the distributions of the dipole for different lifetimes are fitted with a function which is then used as calibration curve. The lifetime is extracted from the comparison of the average of the dipole data distribution with the calibration curve.

It is important to note that what is actually measured is, in first approximation, the product $\tau_{b} \times \Gamma^{b b} / \Gamma^{\text {had }}$, where the fraction of $b \bar{b}$ events in hadronic Z decays, $\Gamma^{b b} / \Gamma^{\text {had }}$, is taken from Standard Model calculation.

If the exclusive $b$ lifetimes are different, the lifetimes of the different species will be weighted with their relative production rates and, to first order, with their average charged multiplicities.

\subsection{Event and track selections}

The event and track selections are not designed to enrich the sample with $b \bar{b}$ events but to choose good quality tracks; they leave the flavour composition essentially unchanged.

Only data recorded with the VDET operational are considered and the standard ALEPH hadronic event selection is applied. As discussed in detail in [15], an event is selected if it has a total charged energy in excess of $15 \mathrm{GeV}$, the sphericity axis lies between $35^{\circ} \leq \Theta_{s} \leq 145^{\circ}$ and has at least five good tracks. A good track has a polar angle with respect to the beam between $20^{\circ}$ and $160^{\circ}$, at least four TPC hits, a transverse momentum $p_{T}$ larger than $0.2 \mathrm{GeV}$ and originates from a cylindrical region around the origin with radius $3 \mathrm{~cm}$ and half-length $5 \mathrm{~cm}$. After this preselection of hadronic events, an additional set of cuts is applied to ensure that the events are two-jet-like and well contained in the tracking detectors: the polar angle of the thrust axis calculated using an energy flow algorithm is required to be between $50^{\circ} \leq \Theta_{T} \leq 130^{\circ}$ and the thrust to be larger than 0.8 .

Track selection criteria are applied to the remaining events: pairs of oppositely charged tracks ( $V^{0}$ candidates) are rejected if the invariant mass of the tracks is consistent within $10 \mathrm{MeV}$ with the $K_{S}^{0}$ or $\Lambda^{0}$ mass hypothesis or within $15 \mathrm{MeV}$ with the hypothesis of $\gamma \rightarrow e^{+} e^{-}$conversions and the distance $d l$ between the reconstructed $V^{0}$ vertex and the beam spot center is larger than $6 \mathrm{~cm}$; the remaining tracks are required to have a momentum larger than $1.0 \mathrm{GeV}$, a $\chi^{2}$ per degree of freedom of the helix fit less than four, at least four hits in the ITC, eight hits in the TPC, one $r$ - $\phi$ and one $r$ - $z$ hit in the VDET. The tracks and the thrust axis are projected onto the $r$ - $\phi$ plane, the thrust axis is positioned on the beam spot center and the intersections of the tracks with it are calculated; the impact parameter $d_{0}$ of a track with respect to the beam spot center is required to be less than $0.5 \mathrm{~cm}$ 
and the distance $d l$ between the intersection of the track with the axis and the beam spot center to be less than $6 \mathrm{~cm}$. These last cuts are intended to remove decay and conversion background and badly measured tracks. After these cuts at least two tracks are required in each hemisphere.

The fractions of preselected hadronic events passing the cuts for Monte Carlo and data are $52.5 \pm 0.1 \%$ and $50.1 \pm 0.1 \%$, respectively. The small difference between Monte Carlo and data is due to the different number of hits produced in the various chambers. The cuts on the hit numbers do not bias the sample with respect to lifetime, because no path-length-dependent information is used. The cuts on momenta and vertices of long lived particles modify the flavour composition of the sample. The Monte Carlo predicts that the fraction of $b \bar{b}$ events is 0.219 before the cuts and 0.244 after. This effect is taken into account in the calibration procedure.

\subsection{Track weights}

In calculating the dipole, the tracks are weighted with the product of the inverse squared position error $w_{i}$ on the intersection of the track $i$ with the thrust axis multiplied by the rapidity of the track $y_{i}$. When the error on the azimuthal angle of the track is negligible, the weight is $w_{i}=\sin ^{2} \Psi_{i} / \sigma^{2}\left(d_{0 i}\right)$, where $\Psi_{i}$ is the angle between the event axis and the track in the $r-\phi$ plane.

The rapidity of a track is defined as $y_{i}=\frac{1}{2} \ln \left(\frac{E_{i}+p_{i \|}}{E_{i}-p_{i} \|}\right)$, where $p_{i \|}$ is the projection of the momentum of the tracks along the thrust axis and the pion mass is used to calculate the energy. Weighting the tracks with their rapidity gives a longer dipole enhancing the contribution of tracks from $b$ hadrons compared with tracks from fragmentation. The weight distributions for data and Monte Carlo are in good agreement.

\subsection{Axis positioning}

Two techniques, described in the following, are exploited to position the thrust axis in the $r$ - $\phi$ plane. It can be positioned on the beam spot center determined for every fill (beam spot) or on the event vertex determined on an event by event basis taking into account the beam spot information (event vertex).

The event vertex technique has the smaller statistical and systematic errors and is the one which is adopted; the other is used to estimate the systematic errors due to tracking.

\section{The dipole and lifetime measurement}

After having positioned the axis according to one of the previously described prescriptions, the intersection points $x_{i}$ are recalculated. The dipole $\rho$ and its weight $w_{\rho}$ are computed in the $r-\phi$ plane and then projected along the thrust axis

$$
\rho=\left(\frac{\sum_{i \in A} x_{i} w_{i} y_{i}}{\sum_{i \in A} w_{i} y_{i}}-\frac{\sum_{j \in B} x_{j} w_{j} y_{j}}{\sum_{j \in B} w_{j} y_{j}}\right) / \sin \Theta_{T},
$$




$$
w_{\rho}=\left(\frac{1}{\sum_{i \in A} w_{i} y_{i}}+\frac{1}{\sum_{j \in B} w_{j} y_{j}}\right)^{-1} \times \sin ^{2} \Theta_{T},
$$

where $\Theta_{T}$ is the polar angle of the thrust axis and $A$ and $B$ correspond to the two hemispheres.

The Monte Carlo sample has been produced with $\tau_{b}=1.50 \mathrm{ps}$ equal for all the $b$ species and with the Standard Model $\mathrm{Z}$ branching ratios for $m_{\text {top }}=100 \mathrm{GeV}$ and $m_{\text {Higgs }}=100 \mathrm{GeV}: \Gamma^{b b} / \Gamma^{\text {had }}=0.218$ and $\Gamma^{b b} / \Gamma^{\text {had }}=0.171$. The uncertainties on these branching ratios due to the unknown values of $m_{\text {top }}$ and $m_{H i g g s}$ are properly accounted for as systematic errors.

The Monte Carlo and data dipole distributions are plotted in Fig. 7; the averages of the data $\rho_{D}$ and Monte Carlo $\rho_{M C}$ dipole distribution are

$$
\rho_{D}=920.1 \pm 8.1 \mu \mathrm{m} \quad \rho_{M C}=915.3 \pm 5.6 \mu \mathrm{m} .
$$

To determine the $b$ lifetime from the average dipole a Monte Carlo calibration curve is used. A sample of $54000 b \bar{b}$ events was simulated with different $b$ lifetimes $\left(\tau_{b}=0.00 \mathrm{ps}, 0.75 \mathrm{ps}, 1.50 \mathrm{ps}, 2.25 \mathrm{ps}\right.$ and $3.00 \mathrm{ps}$ ) and combined with non- $b \bar{b}$ events according to the Standard Model branching ratios to obtain the average dipoles $\rho_{M C}\left(\tau_{b}\right)$. The following parametrization ( $\tau_{b}$ in ps, $\rho$ in $\mu \mathrm{m}$ ) is fitted to the calibration points

$$
\rho_{M C}\left(\tau_{b}\right)=a+b\left(\tau_{b}-1.50\right)\left(1-\exp \left(-c / \tau_{b}\right)\right) .
$$

The fit is shown in Fig. 7 and the fitted parameters are

$$
a=915.3 \pm 5.6 \mu \mathrm{m} \quad b=439.0 \pm 4.3 \mu \mathrm{m} / \mathrm{ps} \quad c=4.31 \pm 0.14 \mathrm{ps} .
$$

The quoted errors are only the statistical errors from the Monte Carlo simulation. The $b$ lifetime obtained using $\rho_{D}$ from Eq.1 and the coefficients in Eq.2 is

$$
\tau_{b}=1.511 \pm 0.022 \mathrm{ps},
$$

where the error is statistical only.

\section{Systematic errors}

The systematic errors can be divided into two categories: those due to the algorithm used and to detector effects and those due to uncertainties in the physics simulation. The latter are estimated by changing the physics simulation parameters influencing the measurement and by studying the effect of the cuts that discriminate between $b$ and non- $b$ components.

In Table 1 the set of cuts used in the standard analysis (Normal) is shown together with alternative sets of cuts on the physics and tracking parameters. The results for all sets of cuts are summarized in Table 2. Taking into account the correlations between the samples, the small differences in the lifetime obtained are compatible with being statistical fluctuations. The largest difference is taken as an estimate of a possible bias introduced by the particular values of the cuts. 
Table 1: The event and track cuts in the options used for systematic checks.

\begin{tabular}{|l|r|r|r|r|r|}
\hline Cut & Normal & Short & Long & $\begin{array}{r}\text { Low } \\
\text { momentum }\end{array}$ & $\begin{array}{r}\text { High } \\
\text { momentum }\end{array}$ \\
\hline Thrust & 0.8 & 0.8 & 0.8 & 0.7 & 0.9 \\
\hline Momentum $(\mathrm{GeV})$ & 1.0 & 1.0 & 1.0 & 0.5 & 1.8 \\
\hline$d_{0}(\mathrm{~cm})$ & 0.5 & 0.3 & 3.0 & 0.5 & 0.5 \\
\hline$d l(\mathrm{~cm})$ & 6.0 & 4.0 & $\infty$ & 6.0 & 6.0 \\
\hline
\end{tabular}

Table 2: Lifetimes and average dipoles for data $\rho_{D}$ and $\rho_{M C}$ for different sets of cuts and positioning techniques.

\begin{tabular}{|l|l|l|l|}
\hline & $\tau_{B}(\mathrm{ps})$ & $\rho_{D}(\mu \mathrm{m})$ & $\rho_{M C}(\mu \mathrm{m})$ \\
\hline Normal & $1.511 \pm 0.022$ & $920.1 \pm 8.1$ & $915.3 \pm 5.6$ \\
\hline No rapidity & $1.483 \pm 0.022$ & $766.5 \pm 7.3$ & $772.7 \pm 5.1$ \\
\hline Beam spot & $1.507 \pm 0.025$ & $909.0 \pm 8.6$ & $906.1 \pm 6.1$ \\
\hline High resolution & $1.479 \pm 0.035$ & $895.9 \pm 11.1$ & $898.3 \pm 10.3$ \\
\hline Short & $1.499 \pm 0.029$ & $862.5 \pm 8.3$ & $863.2 \pm 8.9$ \\
\hline Long & $1.496 \pm 0.028$ & $1127.0 \pm 10.1$ & $1129.1 \pm 11.1$ \\
\hline Low momentum & $1.496 \pm 0.028$ & $815.0 \pm 7.4$ & $816.8 \pm 8.2$ \\
\hline High momentum & $1.507 \pm 0.036$ & $1231.3 \pm 14.5$ & $1227.1 \pm 15.2$ \\
\hline All $V^{0}$ removed & $1.515 \pm 0.032$ & $872.6 \pm 9.8$ & $866.4 \pm 9.0$ \\
\hline
\end{tabular}

\subsection{Systematic errors from tracking and physics simulation}

The $b$ lifetime has also been measured using the alternative positioning technique previously described (Beam spot). This has a negligible effect on the lifetime measurement.

The influence of the resolution simulation is studied by requiring two VDET hits in both $r-\phi$ and $r-z$ views (High resolution). This effect is studied by comparing the negative side of the dipole distribution in data and Monte Carlo. In Fig. 7 data with normal cuts show a slightly worse resolution than the simulation. This discrepancy disappears when using the High resolution cuts.

Further sets of cuts (Low and High momentum, Short and Long dipole) are chosen to decrease and increase, respectively, the relative weight of the tracks coming from $b$ decays versus that of the tracks from hadronization and to remove more or fewer tracks from long-lived particles. In the Low and High momentum sets the flavour composition is changed so that the fraction of $b \bar{b}$ events, equal to 0.244 for the Normal set, is 0.232 and 0.253 , respectively; this tests a possible flavour bias coming from the selection procedure. In addition the normal set is used without the rapidity weighting (No rapidity) and with the $V^{0}$ candidates removed for any $V^{0}$ vertex position (All $V^{0}$ removed). Note, for the Long set no $V^{0}$ is removed.

Among the different sets of cuts the dipole length changes by a factor of 1.6 , while the lifetime is stable within $2 \%$. This indicates that the simulation is adequate for both the hadronization in $b \bar{b}$ and non- $b \bar{b}$ events, including the selection efficiency of 
$b \bar{b}$ events, and the reconstruction of tracks from long-lived particles. The systematic error coming from the uncertainty in both detector effects and physics simulation is taken as $\pm 32 \mathrm{fs}$, the largest deviation from the Normal-cut lifetime among the values in Table 2.

\subsection{Studies of individual error sources}

After this general consistency check of the simulation, the possible physics sources of error are estimated separately. The average dipole for Monte Carlo light quark events $(u \bar{u}, d \bar{d}, s \bar{s})$ is $94.2 \pm 4.0 \mu \mathrm{m}$, that is the contribution from $\gamma$ conversions and decays from long-lived particles. Besides that, the average dipole has three other sources: lifetime of $b$ hadrons $\tau_{b}$, lifetime of charmed hadrons in $c \bar{c}$ events $\tau_{c}^{c c}$, lifetime of charmed hadrons in $b \bar{b}$ events $\tau_{c}^{b b}$. The contribution of $\tau_{b}$ to the average dipole is $71.9 \%$, that of $\tau_{c}^{c c}$ is $8.1 \%$, that of $\tau_{c}^{b b}$ is $7.3 \%$, that of other sources 1 s $12.7 \%$.

For the different sets of cuts from Table 1 , the contribution from sources other than the heavy-flavour lifetimes varies from $6.8 \%$ (Short) to $35.6 \%$ (Long). More specifically, the average dipole for light quark events varies between $45 \mu \mathrm{m}$ (Short) to $309 \mu \mathrm{m}$ (Long). Removing all the tracks compatible with a $\Lambda^{0}, K_{S}^{0}$ or $\gamma$ vertex for all positions of the vertex (All $V^{0}$ removed), changes the dipole for light quark events to $64 \mu \mathrm{m}$. The remaining dipole corresponds to other decays $\left(\pi^{ \pm}, K^{ \pm}, \Sigma^{ \pm}, \ldots\right)$ and to inefficiency in the $V^{0}$ finding procedure. The variation of the lifetime extracted from the data over this range of light quark contribution is shown in Table 2. The error from the light quark contribution uncertainty is therefore included in the overall $32 \mathrm{fs}$ systematic error.

Another check on the quality of the simulation of the momentum and rapidity distributions is obtained by plotting the rapidity distributions for data and Monte Carlo for the Normal and High momentum sets, as shown in Fig. 7. For both sets of cuts, the differences between the Monte Carlo and the data distributions divided by their sums are plotted in Fig. 7 and display good agreement for $y>1$.. The effect of the disagreement on the lifetime for $y<1$. is checked through the High momentum set of cuts, that depletes this region by over $80 \%$ of the tracks.

The other systematic errors can be divided into those related to the charm decay properties, which are common to the contributions from $\tau_{c}^{c c}$ and $\tau_{c}^{b b}$, those specific to the production mechanism of charmed hadrons in $b \bar{b}$ and $c \bar{c}$ events, respectively, and those related to the $b$ hadron production and decay mechanism. The error sources are listed in Table 3. The most relevant error sources are discussed in more detail in the following.

The $b$ fragmentation function has been measured at LEP with and without the assumption of the functional form given in [17]. A value of $\left\langle x_{b}\right\rangle=0.695 \pm 0.015$ that covers the full range of LEP measurements [26, 29] is taken. The error on the $b$ lifetime coming from the $b$ fragmentation includes both varying the parameter of the fragmentation function within its error and using an alternative fragmentation function $x^{\alpha}(1-x)[18]$ tuned to reproduce the measured $\left\langle x_{b}\right\rangle$.

The errors from the uncertainties in the charged multiplicities of charmed and $b$ hadron decays come from inclusive measurements of charged multiplicity distributions at the $\Psi(3770)$ and $\Upsilon(4 s)$ resonances. They are increased to account for the additional production of charmed strange mesons and baryons at the LEP energy. 
Table 3: Systematic errors on the $b$ lifetime.

\begin{tabular}{|l|l|l|l|}
\hline Error source & Error[fs] & Ref. \\
\hline Charm overall & Charm lifetime & 11 & {$[19]$} \\
& Charm charged multiplcity & 18 & {$[20]$} \\
\hline Charm from $c \bar{c}$ & Charm fragmentation & 6 & {$[21]$} \\
& $D^{0}, D^{+}$fractions & 6 & {$[21]$} \\
& $D_{s}$ fraction & 1 & {$[22]$} \\
& Charm baryon fraction & 1 & {$[22]$} \\
\hline Charm from $b \bar{b}$ & Charm spectrum & 24 & {$[24,25]$} \\
& Species abundance & 15 & {$[24]$} \\
\hline$b$ & $b$ fragmentation & 42 & {$[26,29]$} \\
& $B^{0}, B^{+}$fractions & 5 & \\
& $B_{s}$ fraction & 2 & {$[27]$} \\
& Baryon fraction & 8 & {$[27]$} \\
& $b$ charged multiplcity & 37 & {$[28]$} \\
& $\tau_{b}$ differences & 8 & \\
\hline Hadronization & Simulation & 17 & {$[15]$} \\
\hline Cut sensitivity & 32 & \\
\hline Theoretical error $\Gamma^{b b} / \Gamma^{\text {had }}$ & 13 & {$[23]$} \\
\hline Theoretical error $\Gamma^{c c} / \Gamma^{\text {had }}$ & 1 & {$[23]$} \\
\hline
\end{tabular}

The theoretical uncertainties on the branching ratios $\Gamma^{c c} / \Gamma^{\text {had }}$ and $\Gamma^{b b} / \Gamma^{\text {had }}$ are also included. Summing quadratically the systematic errors from Table 3 the measurement in Eq. 3 becomes

$$
\tau_{b}=1.511 \pm 0.022(\text { stat }) \pm 0.078(\text { syst }) \mathrm{ps}
$$

\section{Comparison with the previous ALEPH $b$ life- time measurement with leptons}

ALEPH has published an analysis on the inclusive $b$ lifetime in [7] using the impact parameter distribution of high $p, p_{T}$ leptons. The result is $\tau_{b}=1.49 \pm 0.03 \pm 0.06 \mathrm{ps}$, where the first error is statistical and the second systematic.

If the lifetimes of the different $b$ hadron species are different, the two analyses are measuring different quantities. In first approximation, the lepton analysis measures the average lifetime weighted with the semileptonic branching ratios, while the dipole analysis measures the average lifetime weighted with the average charged multiplicities. Using a simplified model with only two $b$ hadron species with $20 \%$ relative difference in lifetimes and $20 \%$ in charged multiplicities, both the lepton weighted average and the charged multiplicity weighted average differ from the true average by less than $1 \%$; therefore the quantities measured are effectively, if not conceptually, the same and can be compared. 
The statistical and systematic errors of the two measurements are almost completely uncorrelated; the average is

$$
\tau_{b}=1.50 \pm 0.02 \pm 0.05 \mathrm{ps} .
$$

\section{Conclusion}

The lifetime of $b$ hadrons is measured using the dipole method. This method does not depend on any $b$ enrichment scheme and has different systematic errors from lifetime measurements through the impact parameters distribution of high $p, p_{T}$ leptons.

The result obtained using the Standard Model values for $\Gamma^{b b} / \Gamma^{\text {had }}$ and $\Gamma^{c c} / \Gamma^{\text {had }}$ is

$$
\tau_{b}=1.51 \pm 0.08 \mathrm{ps}
$$

Averaging it with the previous ALEPH $b$ lifetime measurement using the lepton impact parameter distribution, the combined result is

$$
\tau_{b}=1.50 \pm 0.05 \mathrm{ps}
$$

\section{Acknowledgement}

We are indebted to our colleagues in the accelerator divisions for the good performance of the LEP storage ring. We thank also the engineers and technicians of all our institutions for their support in constructing ALEPH. Those of us from non-member countries thank CERN for its hospitality.

\section{References}

[1] MAC Collab., W.W. Ash et al., Phys. Rev. Lett. 58 (1987) 640.

[2] HRS Collab., J.M. Brom et al., Phys. Lett. B195 (1987) 301.

[3] DELCO Collab., D.A. Klem et al., Phys. Rev. D37 (1988) 41.

[4] MARK II Collab., R.A. Ong et al., Phys. Rev. Lett. 62 (1989) 1236.

[5] TASSO Collab., W. Braunschweig et al., Z. Phys. C44 (1989) 1.

[6] JADE Collab., J. Hagemann et al., Z. Phys. C48 (1990) 401.

[7] ALEPH Collab., D. Buskulic et al., Phys. Lett. B295 (1992) 174.

[8] DELPHI Collab., P. Abreau et al., Z. Phys. C53 (1992) 567.

[9] L3 Collab., B. Adeva et al., Phys. Lett. B270 (1991) 111. 
[10] OPAL Collab., P.D. Acton et al., CERN-PPE/93-92, (June 1993). Submitted to Z. Phys. C.

[11] K.U. Pösneker, Doktor Arbeit, Universität Hamburg (1987) (unpublished).

[12] ALEPH Collab., D. Decamp et al., Nucl. Instr. \& Meth. A294 (1990) 121.

[13] G. Batignani et al., Conference Record of the 1991 IEEE Nucl. Science Symp., 1991, Santa Fe, New Mexico, USA, Vol 1, 438.

[14] ALEPH Collab., D. Decamp et al., Nucl. Instr. \& Meth. A323 (1992) 213.

[15] ALEPH Collab., D. Decamp et al., Phys. Lett. B273 (1991) 181.

[16] L3 Collab., B. Adeva et al., Phys. Lett. B261 (1991) 177.

[17] C. Peterson et al., Phys. Rev. D27 (1983) 105.

[18] V.G. Kartvelishvili et al., Phys. Lett. B78 (1978) 615.

[19] K. Hikasa et al., Review of particle properties, Phys. Rev. D45, Part 2 (1992) 1.

[20] MARK I Collab., V. Vuillemin et al., Phys. Rev. Lett. 41 (1978) 1149.

MARK II Collab., R.H. Schindler et al., Phys. Rev. D24 (1981) 78.

MARK III Collab., D. Coffman et al., Phys. Lett. B263 (1991) 135.

LEBC-EHS Collab., M. Aguillar-Benitez et al., Z. Phys. C36 (1987) 551.

[21] ALEPH Collab., D. Decamp et al., Phys. Lett. B266 (1991) 218. Production of Charmed Mesons in Z decays, paper in preparation.

[22] ALEPH Collab., D. Buskulic et al., Phys. Lett. B284 (1992) 177.

[23] J.H. Kühn and P.M. Zerwas in: Z Physics at LEP1, CERN 89-08 Vol. 1, Sept. 1989, pp. 271-275, Edited by G. Altarelli, R. Kleiss and C. Verzegnassi.

[24] M. Ali and P. Söding, High Energy Electron-Positron Physics, World Scientific, pp. 273-357.

[25] CLEO Collab., G. Crawford et al., Phys. Rev. D45 (1992) 752.

[26] ALEPH Collab., D. Buskulic et al., Heavy Flavour Quark Production and Decay Using prompt Leptons in the ALEPH Detector, paper in preparation.

[27] ALEPH Collab., D. Buskulic et al., Phys. Lett. B294 (1992) 45. OPAL Collab., P.D. Acton et al., CERN-PPE/92-144, Submitted to Phys. Lett. B.

[28] B.Gittelman and S.Stone, CLNS-81/87 (1987).

M. Ali and P. Söding, High Energy Electron-Positron Physics, World Scientific, p. 282.

ARGUS Collab., H. Albrecht et al., Z. Phys. C54 (1992) 13. 
[29] OPAL Collab., M.Z. Akrawy et al., Phys. Lett. B263 (1991) 311. DELPHI Collab., P. Abreau et al., Z. Phys. C56 (1992) 47.

L3 Collab., B. Adeva et al., Phys. Lett. B261 (1991) 177.

L3 Collab., B. Adeva et al., Phys. Lett. B288 (1992) 412. 


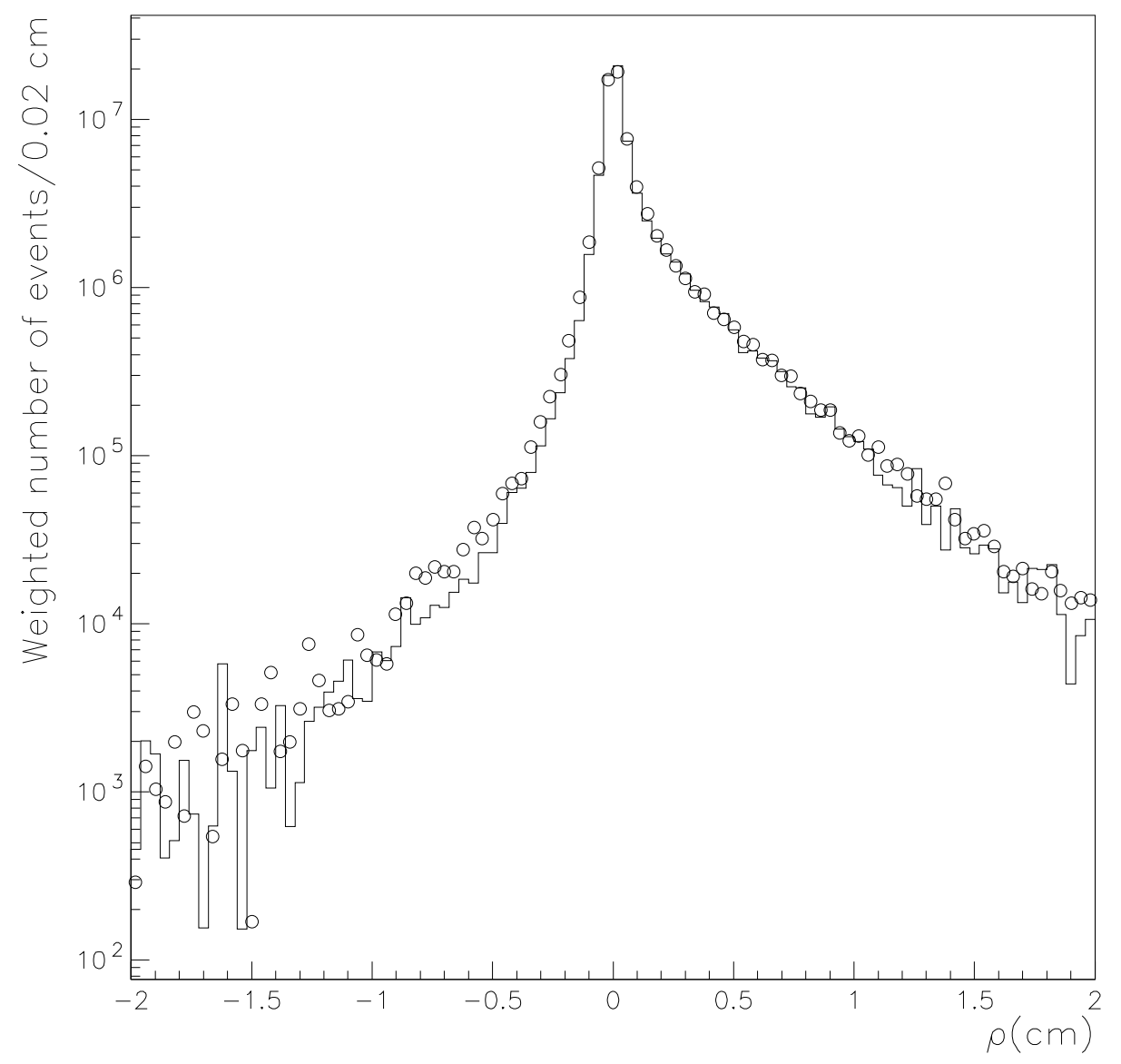

Figure 1: The dipole distributions for Monte Carlo (solid) and data (points). The generated Monte Carlo $b$ lifetime was 1.50 ps. 


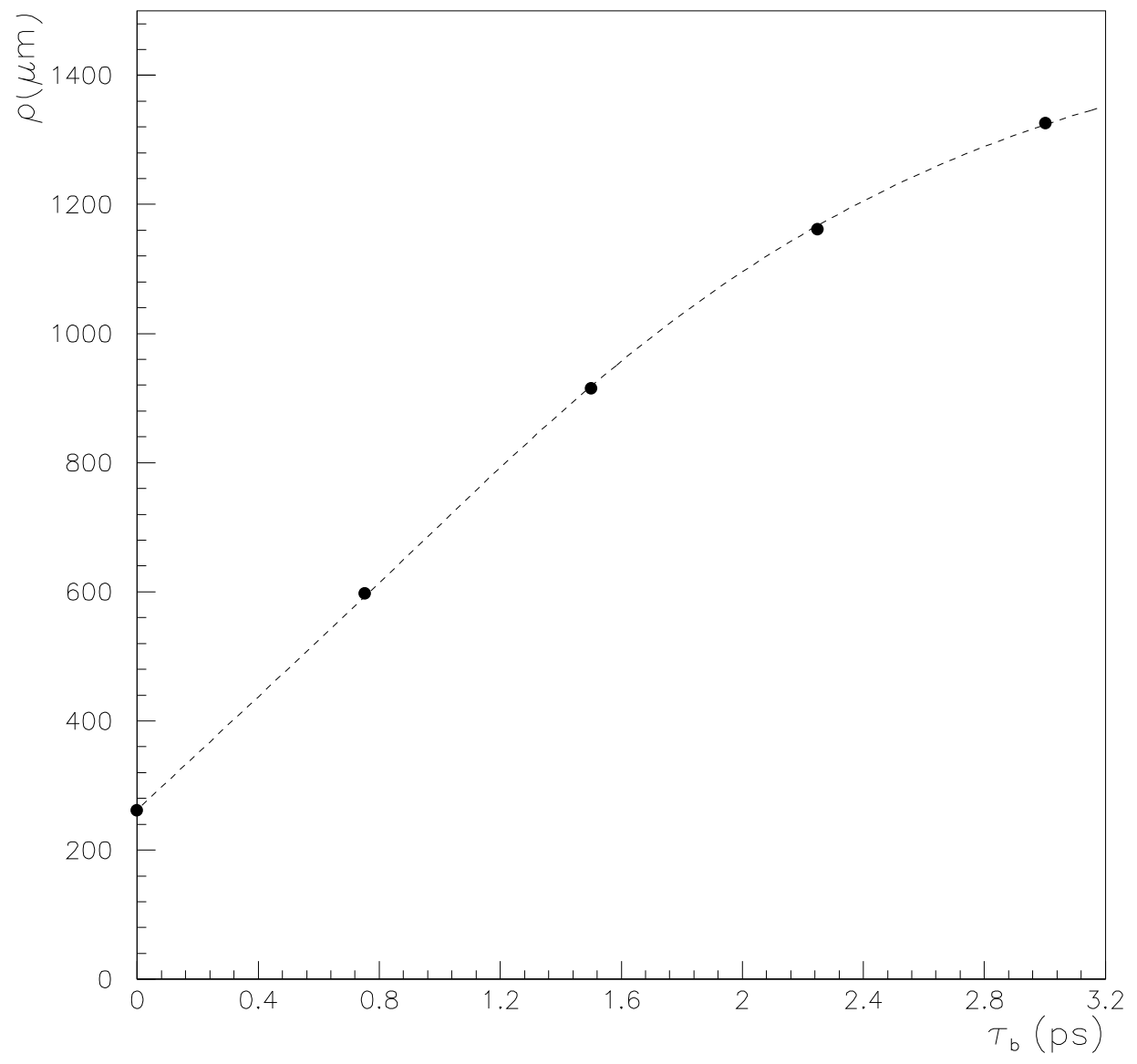

Figure 2: Monte Carlo dipole with the normal selection versus $\tau_{b}$. The dashed line is the result of a parametrization. 

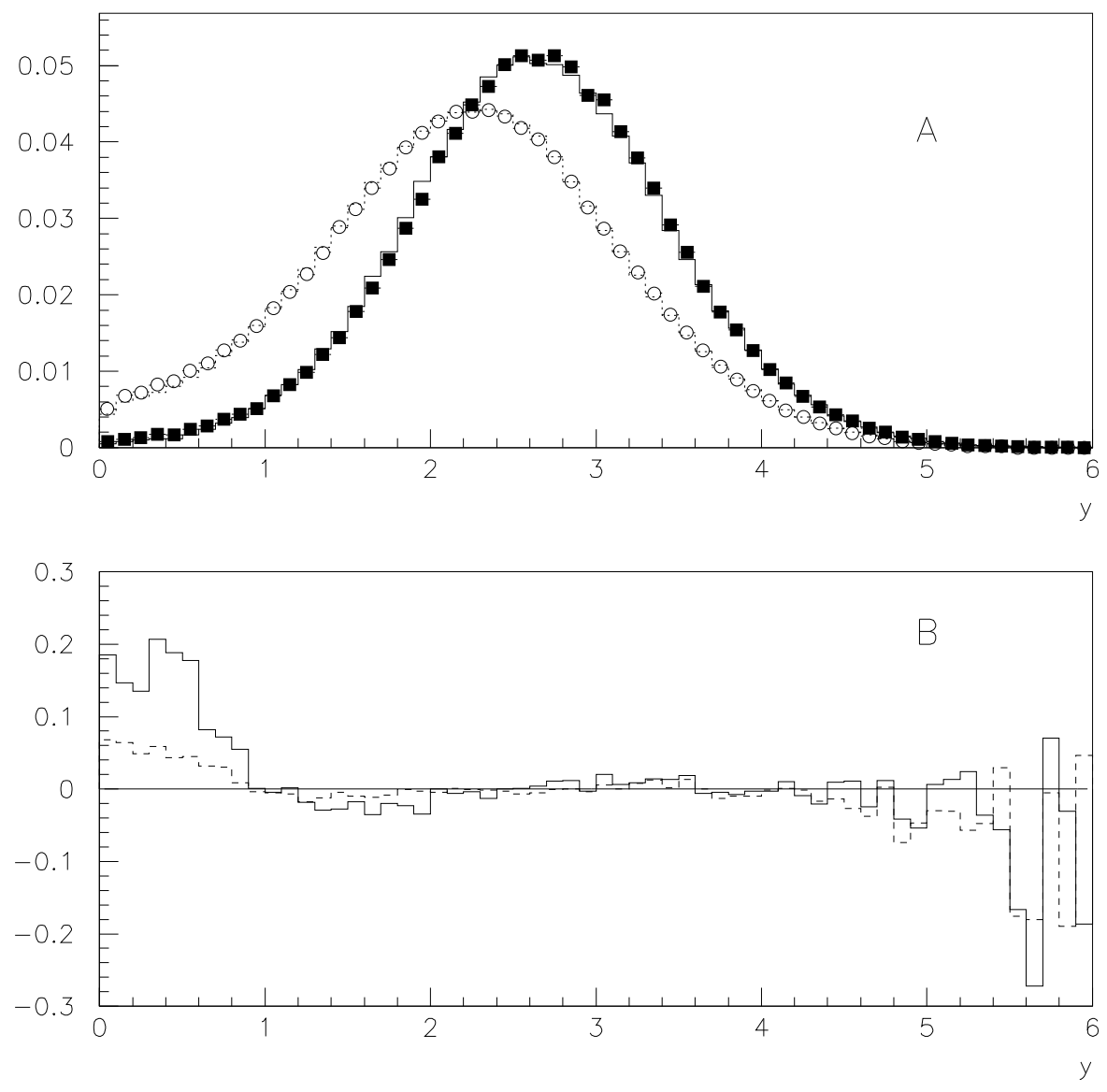

Figure 3: A) Rapidity distributions of Monte Carlo (dashed line) and data (hollow circles) for Normal cuts and of Monte Carlo (solid line) and data (black squares) for High momentum cuts. B) Normalized differences (Monte Carlo - Data)/(Monte Carlo + Data) for Normal (dashed line) and High momentum (solid line) cuts. 